Pathologe 2008 · [Suppl 2] 29:141-144

DOI 10.1007/s00292-008-1077-0

Online publiziert: 28 . September 2008

(c) Springer Medizin Verlag 2008

\author{
H. Regele \\ Klinisches Institut für Pathologie, Medizinische Universität Wien, Österreich
}

\title{
Die Rolle von Endothelzellen in der Transplantatabstoßung
}

munhistochemische Nachweis des Komplementspaltproduktes $\mathrm{C}_{4} \mathrm{~d}$ der wichtigste diagnostische Befund ist. Zwar sind mit histologischen Techniken kaum detaillierte Analysen der immunologischen Mechanismen einer Abstoßungsreaktion möglich, jedoch können morphologische Befunde sehr wohl Anstoß und Grundlage für weiterführende Untersuchungen sein. So wirft z. B. die Ablagerung von $\mathrm{C}_{4} \mathrm{~d}$ am kapillären Endothel eines Transplantats ohne gleichzeitigen Nachweis zirkulierender Anti-HLA- („human leucocyte antigen-")AK die Frage nach AK anderer Spezifität auf.

\section{Die Rolle von non-HLA-antiendothelialen AK nach Nierentransplantation}

Die Frage nach derartigen AK ist deshalb von klinischer Relevanz, weil sich gegenwärtige (serologische) Untersuchungsmethoden in der Vorbereitung zur und in der Betreuung nach Transplantation fast nur auf HLA-spezifische AK konzentrieren und $\mathrm{AK}$ anderer Spezifität daher unentdeckt bleiben könnten. Neben dem Befund einer C4d-positiven Anti-HLAAK-Abstoßung legt auch die Beobachtung von Abstoßungsprozessen bei HLAidenten Spender-Empfänger-Paarungen eine mögliche Rolle von Non-HLA-AK nahe. Es existiert tatsächlich eine Vielzahl von Berichten über nicht gegen HLA gerichtete antiendotheliale $\mathrm{AK}$, die einerseits bei Autoimmunerkrankungen beobachtet wurden [1], andererseits auch bei Transplantatempfängern nachweisbar waren und mit einem ungünstigen klinischen Verlauf nach Transplantation assoziiert waren. Dabei handelt es sich jedoch häufig um Fallberichte, und der Nachweis der AK beruhte fast immer auf nichtstandardisierten Tests, die häufig auch keine verlässliche Charakterisierung der involvierten Antigene erlauben [10, 13, 17].

In den 1970er und 1980er Jahren beschäftigten sich mehrere Gruppen mit der AK-Reaktivität gegen Antigene, die auf Endothelzellen und Monozyten exprimiert $[2,6,8]$ und somit konventionellen Detektionmethoden unzugänglich sind. Eine umfassende Charakterisierung dieser Antigene wurde jedoch nicht durchgeführt, und die verfügbaren Daten lassen vermuten, dass es sich nicht um ein einheitliches Antigensystem handelt. In jüngerer Vergangenheit gelang es jedoch, einige Zielantigene von Non-HLA-AK in Nierentransplantatempfängern näher zu charakterisieren. So konnte die Arbeitsgruppe um D. Dragun aus Berlin nachweisen, dass aktivierende AK gegen den Angiotensin-II-Typ-1-Rezeptor mit persistierender vaskulärer Abstoßung und maligner Hypertension assoziiert sind [4].

Das in den letzten Jahren am umfassendsten untersuchte Non-HLA-Antigensystem ist MICA („MHC class I-related chain $\left.A^{\prime \prime}\right)$. MICA-Gene kodieren in der Nähe des HLA-Klasse-Ib-Locus auf Chromosom 6 für Moleküle, die auf der Oberfläche von Endothelzellen (nicht mit Beta-2-Mikroglobulin assoziiert) exprimiert sind, jedoch auf Lymphozyten fehlen. Zwei große unabhängige Studien an 1329 [14] bzw. 1910 [18] Nierentransplantatempfängern zeigten ein signifikant vermindertes Transplantatüberleben in Empfängern mit entweder präformierten [18] oder post transplantationem nachgewiesenen [14] Anti-MICA-AK.

Zur Integration der oben genannten Erkenntnisse in die klinische Praxis würden allgemein verfügbare standardisier- 
te Tests benötigt, die jedoch gegenwärtig noch fehlen. Eine mögliche Strategie zur Detektion von Non-HLA-AK wäre die Verwendung von Endothelzellen statt Lymphozyten für „Crossmatch-Tests“ und andere serologische Untersuchungen. Dieser Zugang wurde auch von mehreren Gruppen gewählt, in deren Studien in der Regel immortalisierte endotheliale Zellen [10] oder Endothelzellen aus humanen Nabelschnurvenen (HUVEC; [17]), nicht aber Zellen des Spenders (wie bei konventionellen Crossmatch-Tests) verwendet wurden. Die Tatsache, dass sich diese Zellen hinsichtlich ihres HLA-Typs, aber auch in Bezug auf Nicht-MHC-Antigene von den Spenderendothelzellen unterscheiden, kann die Relevanz derartiger Untersuchungsmethoden einschränken.

Um eine spenderspezifische antiendotheliale AK-Reaktivität direkt bestimmen zu können, haben wir daher Spenderendothelzellen (makrovaskuläre aus der Aorta und mikrovaskuläre aus der Nebenniere) isoliert und sie nach Inkubation mit Empfängerserum mittels Flow-Zytometrie auf gebundene Empfängerantikörper untersucht. Um eine Unterscheidung zwischen Anti-HLA- und Non-HLA-AK zu ermöglichen, wurden alle Sera auch per T-ZellCrossmatch und mittels HLA-beschichteter „FloPRA@ beads“ (OneLambda) untersucht. Ein positives EndothelzellCrossmatch bei gleichzeitig negativen Resultaten im T-Zell-Crossmatch und im FloPRA@-Test wurde als Nachweis von Non-HLA-AK angesehen. In 8 von 75 untersuchten Patienten konnten tatsächlich Non-HLA-AK (ohne gleichzeitige AntiHLA-Reaktivität) festgestellt werden. Der Nachweis dieser AK war allerdings weder mit einer $\mathrm{C}_{4} \mathrm{~d}$-Ablagerung in Transplantatbiopsien noch mit einer klinisch nachweisbaren Verschlechterung der Transplantatfunktion assoziiert (unveröffentlichte Beobachtung). Aus diesen Ergebnissen kann daher keine Notwendigkeit für eine die klassischen Crossmatch-Untersuchungen ergänzende, systematische Suche nach Non-HLA-AK abgeleitet werden. Es muss jedoch in Betracht gezogen werden, dass eine (potenziell auch klinisch relevante) Non-HLA-AK-Reaktivität gleichzeitig mit Anti-HLA-AK besteht und von diesen in unserem Testsystem ,verdeckt“ werden könnte.

\section{Mechanismen AK-mediierter Transplantatschädigung}

Obwohl die AMR eines Nierentranplantats ein diagnostisch und klinisch gut definiertes Geschehen ist, weiß man erstaunlich wenig über die molekularen Mechanismen des oft schweren, jedoch unter adäquater Therapie auch reversiblen Funktionsverlustes. Auch bietet die genaue Analyse der in Transplantatbiopsien zu beobachtenden Veränderungen wesentliche Anhaltspunkte und Anregungen zur Entwicklung pathogenetischer Konzepte. Einerseits findet man das beeindruckende Bild der so genannten (hy)perakuten Abstoßung, die bei gegen Spenderantigene vorsensibilisierten Empfängern zu einem massiven AK- und komplementvermittelten Endothel- und Gefäßwandschaden führt. Die massiven arteriellen Läsionen mit begleitender Thrombose führen hier binnen weniger Stunden nach Transplantation zu einem irreversiblen ischämischen Organschaden. Dieses Bild war allerdings fast nur bei vorsensibilisierten Empfängern zu beobachten und ist heute durch die nahezu lückenlose CrossmatchUntersuchung eine Rarität geworden.

Andererseits stehen bei der typischerweise in den ersten Wochen nach Transplantation auftretenden akuten AMR häufig relativ milde histomorphologische Läsionen im Gegensatz zu einer meist hochgradigen Nierenfunktionseinschränkung. Meist ist hier das histologische Bild von intravasaler Immunzellakkumulation in Glomerula und peritubulären Kapillaren gekennzeichnet, und nur selten finden sich höhergradige Gefäßläsionen mit Thrombose. Im Unterschied zur hyperakuten Abstoßung erschließt sich hier der Schädigungsmechanismus nicht aus den histologisch nachweisbaren Gewebsläsionen. Die Reversibilität des Organschadens durch die Entfernung zirkulierender AK mittels extrakorporaler Verfahren (Immunadsorption, Plasmapherese) spricht jedoch deutlich für eine wichtige pathogenetische Rolle von Anti-HLA(und evtl. anderen) Alloantikörper.

Sowohl Versuche mit kultivierten Endothelzellen als auch tierexperimentelle Daten legen nahe, dass neben der klassischen AK- und komplementvermittelten direkten Zellzerstörung (die offen- bar bei hyperakuter Abstoßung vorliegt) AK mittels anderer Mechanismen einen Gewebsschaden mediieren können. So wurde in vitro eine durch antiendotheliale AK (AEA) von Patienten mit Lupus erythematodes induzierte so genannte proinflammatorische Endothelzellaktivierung [9] beobachtet. Ähnliche Befunde wurden auch für Autoantikörper bei anderen Autoimmunerkrankungen sowie für Anti-HLA-AK erhoben [15]. Die potenziell proinflammatorische Wirkung von AEA wird weiterhin durch eine AK-induzierte Aktivierung des Transkriptionsfaktors „nuclear factor kappa B“ (NF-кB; [11]) nahegelegt, da dieser Transkriptionsfaktor eine zentrale Rolle bei der Expression einer Vielzahl proinflammatorischer Gene spielt [3]. Eine derartige durch AEA vermittelte funktionelle Veränderung der Endothelzellen würde auch gut zur Akkumulation von Immunzellen in renalen Kapillaren passen, die häufig bei $\mathrm{C}_{4} \mathrm{~d}$-positiver AMR beobachtet wird.

Neben der Induktion eines proinflammatorischen Phänotyps könnten AEA in der AMR auch durch die Förderung prokoagulatorischer Eigenschaften von Endothelzellen oder durch eine gesteigerte apoptotische Aktivität wirksam sein. Diese Mechanismen wurden zwar in verschiedenen experimentellen Systemen nachgewiesen, ihre konkrete Rolle in der AMR humaner Nierentransplantate ist jedoch ungewiss.

\section{Experimentelle Modelle der AK-mediierten Abstoßung}

Will man die Mechanismen der AMR exakt dissezieren, erweisen sich konventionelle tierexperimentelle Transplantationsmodelle als wenig geeignet, da es nahezu unmöglich ist, den Beitrag AK-abhängiger Immunmechanismen von zellmediierten Prozessen zu trennen. Aus diesem Grunde wurden Nierentransplantationsmodelle in immundefizienten Tieren entwickelt, um so eine isolierte und kontrollierte AMR im lebenden Organismus zu simulieren. Die Gruppe um P. Halloran hat in einem sehr eleganten Modell rag-/-_"Knock-outMäuse“ als Empfänger von Nierentransplantaten ausgewählt [12], da diese Tiere weder T-Zellen noch B-Zellen besitzen und somit zu keiner adaptiven Immunant- 
wort bzw. Transplantatabstoßung in der Lage sind. Durch Injektion eines komplementaktivierenden AK gegen ein MHCMolekül der Spendertiere wurde eine Situation wie zu Beginn einer klinischen AMR eines Nierentransplantats simuliert, um den klinischen Verlauf und auch die molekulare Reaktion der Endothelzellen mittels DNA-Microarray zu untersuchen. In der Tat banden die Antikörper an die renalen Endothelzellen und führten zur Komplementaktivierung mit endothelialer $\mathrm{C}_{4} \mathrm{~d}$-Ablagerung und damit zu einem Bild wie bei humaner AMR. Verwunderlicherweise entwickelten die Tiere jedoch keine weiteren Zeichen einer Abstoßung und zeigten auch keine Transplantatdysfunktion. Es konnte auch keine veränderte Expression endothelialer Gene beobachtet werden, die einen Hinweis auf eine spezifische Reaktion der Endothelzellen auf AK-Bindung und Komplementaktivierung geben könnte [12].

Um die molekulare Antwort von humanen Endothelzellen auf die Bindung von Anti-HLA-AK mit nachfolgender Komplementaktivierung zu untersuchen, inkubierten wir mikrovaskuläre Endothelzellen mit aus Nierentransplantatempfängern isolierten komplementaktivierenden Alloantikörpern und führten ebenfalls eine Genexpressionsanalyse mittels DNA-Microarray (Affymetrix GeneChip) durch. Während die als Positivkontrolle eingesetzte Stimulation der Endothelzellen mit Zytokinen (Interleukin- $1 \beta$, Tumor-Nekrose-Faktor- $\alpha$, Interferon- $\gamma$ ) eine massiv gesteigerte Genexpression mit dem erwarteten proinflammatotrischen Muster induzierte, konnte bei den mit AK und Komplement beladenen Zellen kein spezifisches Genexpressionsmuster beobachtet werden (unveröffentlichte Beobachtung).

Eine Arbeitsgruppe aus Hannover wählte einen ähnlichen Ansatz wie die Gruppe von P. Halloran und transplantierte Nieren in athymische Nacktratten, um ebenfalls die Effekte einer spontanen Transplantatabstoßung auszublenden [7]. Wie im oben beschriebenen Modell wurde ein komplementaktivierender AK infundiert. In allen AK-infundierten Tieren entwickelte sich etwa 2 Monate nach Transplantation eine Proteinurie und in den meisten Tieren auch eine Nierenin-

Pathologe 2008 · [Suppl 2] 29:141-144 DOI 10.1007/s00292-008-1077-0

(c) Springer Medizin Verlag 2008

\section{H. Regele \\ Die Rolle von Endothelzellen in der Transplantatabstoßung}

\section{Zusammenfassung}

Endothelzellen spielen eine zentrale Rol-

le in der Transplantatabstoßung. Sie sind einerseits Ziel von Immunreaktionen und nehmen andererseits als aktive Partner an der Attraktion und Extravasation von Immunzellen teil. Diese Mechanismen werden auch in Transplantatbiopsien offensichtlich, bei denen einerseits die antikörpermediierte Komplementablagerung am Endothel und andererseits auch die damit assoziierte luminale Immunzellansammlung nachgewiesen werden kann. Obwohl HLA-Moleküle (in AB0kompatiblen Transplantaten) die unbestritten größte Bedeutung als Zielantigene haben, wird auch ein Beitrag von gegen andere Antigene gerichteten Antikörpern (AK) postuliert und für MICA- (,MHC class I-related chain $A^{\prime \prime}$-) und Angiotensin-II-Typ-1-Rezeptor auch überzeugend nachgewiesen. Die klinische Applikation dieser Erkenntnisse wird jedoch durch die mangelnde Verfügbarkeit von standardisierten Tests behindert. Die kausale Rolle von AK in der Transplantatabstoßung ist unbestritten, die molekularen Mechanismen der Transplantatdysfunktion sind jedoch weitgehend unbekannt. Es wurde zwar gezeigt, dass antiendotheliale AK verschiedener Spezifität in vitro Endothelzellen aktivieren können, allerdings konnte eine derartige endotheliale Reaktion in rezenten Untersuchungen in immunzelldefizienten Tiermodellen und In-vitro-Ansätzen (ohne Beteiligung von Immunzellen) nicht nachgewiesen werden. Dies könnte bedeuten, dass in der Effektorphase der AK-mediierten Abstoßung nicht eine direkte Schädigung/Aktivierung der Endothelzellen im Vordergrund steht, sondern eine (evtl. komplementinduzierte) Rekrutierung von Immunzellen an den Ort des Geschehens zumindest in der Frühphase der AK-mediierten Abstoßung entscheidend ist.

\section{Schlüsselwörter}

Transplantatbiopsie · Antikörpermediierte Abstoßung · Nierentransplantation · Alloantikörper · Endothelzellen

\section{The role of endothelial cells in allograft rejection}

\section{Abstract}

Endothelial cells (EC) are crucially involved in allograft rejection. They are prime targets of alloreactivity but also key players in the recruitment and extravasation of immune cells. These mechanisms also become clear in allograft biopsies with antibody-mediated complement deposition on EC and associated intracapillary accumulation of immune cells. HLA molecules are the most prominent targets of alloantibodies in ABO compatible transplantation. Clinically relevant antibodies against other antigens such as MICA ( $M H C$ class I-related chain A) or the angiotensin II Type-1 receptor could also be convincingly demonstrated. The lack of generally available diagnostic tests for such non-HLA antibodies hampers their introduction into clinical practice. Alloantibodies undoubtedly cause allograft rejection. However, our knowledge of the molecular mechanisms underlying graft dysfunction in antibody-media- ted rejection (AMR) is still fragmentary. Activation of EC by anti-endothelial cell antibodies was demonstrated in several experimental systems. Recent animal studies employing immune cell deficient transplant recipients or in-vitro assays, however, failed to demonstrate an immediate response of EC upon antibody binding and complement activation. It might therefore be considered that direct antibody- or complement-mediated EC damage is not necessarily the leading event in acute AMR. Antibody-and/or complement-induced recruitment of immune cells might rather be of crucial importance at least in the early phases of AMR.

\section{Keywords}

Allograft biopsy - Antibody-mediated rejection - Renal transplantation - Alloantibodies - Endothelial cells 
suffizienz. In der Histologie wurde ein Bild ähnlich wie bei chronisch humoraler Transplantatabstoßung beobachtet: chronische Transplantatglomerulopathie und interstitielle Fibrose mit Tubulusatrophie. Da diese Studie leider keine Analyse der frühen Läsionen beinhaltete, kann kein direkter Vergleich mit den oben genannten Untersuchungen vorgenommen werden, weil bei diesen wiederum keine Langzeitbeobachtungen durchgeführt wurden.

Auf den ersten Blick scheint das Auftreten der schweren Läsionen im Rattenmodell im Widerspruch zum Fehlen einer endothelialen Antwort in den erstgenannten Studien zu stehen. Eine mögliche Erklärung für diese Befunde wäre jedoch, dass die akuten Läsionen der AMR nicht primär durch eine endotheliale Antwort initiiert werden, sondern dass möglicherweise eine direkte Interaktion zwischen gebundenen AK bzw. Komplementfaktoren und Immunzellen essenziell für die Entwicklung der in Transplantatbiopsien beobachteten Läsionen ist. Eine direkt durch AK und Komplement (ohne zelluläre Beteiligung) verursachte $\mathrm{Ge}$ websschädigung könnte auf Basis der Befunde aus dem Rattenmodell als ein langsam verlaufender Prozess vermutet werden, der auch auf anderen molekularen Mechanismen beruht.

\section{Fazit für die Praxis}

\section{HLA-Moleküle haben die unbestritten} größte Bedeutung als Zielantigene der AMR. Es wurde jedoch auch ein Beitrag von gegen andere Antigene gerichteten AK wie z. B. für MICA und Angiotensin-IITyp-1-Rezeptor nachgewiesen. Die klinische Anwendung dieser Erkenntnisse wird jedoch durch die noch mangelnde Verfügbarkeit von standardisierten Tests behindert.

Obwohl die zentrale pathogenetische Rolle von AK in der AMR unbestritten ist, sind ihre molekularen Mechanismen noch unklar. Rezente Untersuchungen legen nahe, dass in der Effektorphase der AK-mediierten Abstoßung möglicherweise nicht eine direkte Schädigung/Aktivierung der Endothelzellen im Vordergrund steht, sondern die (evtl. komplementinduzierte) Rekrutierung von Immunzellen an den Ort des Geschehens zumindest in der Frühphase der AK-mediierten AbstoBung entscheidend sein könnte.

\section{Korrespondenzadresse \\ Dr. H. Regele \\ Klinisches Institut für Pathologie, \\ Medizinische Universität Wien \\ Währinger Gürtel 18-20, 1090 Wien \\ Österreich \\ heinz.regele@meduniwien.ac.at}

Interessenkonflikt. Der korrespondierende Autor gibt an, dass kein Interessenkonflikt besteht.

\section{Literatur}

1. Belizna C, Duijvestijn A, Hamidou M, Cohen Tervaert JW (2006) Antiendothelial cell antibodies in vasculitis and connective tissue disease. Ann Rheum Dis 65: 1545-1550

2. Cerilli J, Brasile L, Galouzis T et al. (1985) The vascular endothelial cell antigen system. Transplantation 39: 286-289

3. Denk A, Goebeler M, Schmid S et al. (2001) Activation of NF-kappa B via the lkappa B kinase complex is both essential and sufficient for proinflammatory gene expression in primary endothelial cells. J Biol Chem 276: 28451-28458

4. Dragun D, Muller DN, Brasen JH et al. (2005) Angiotensin II type 1-receptor activating antibodies in renal-allograft rejection. N Engl J Med 352: 558569

5. Fahim T, Bohmig GA, Exner M (2007) The cellular lesion of humoral rejection: predominant recruitment of monocytes to peritubular and glomerular capillaries. Am J Transplant 7: 385-393

6. Joyce S, Flye MW, MohanakumarT (1988) Characterization of kidney cell-specific, non-major histocompatibility complex alloantigen using antibodies eluted from rejected human renal allografts. Transplantation 46: 362-369

7. Koch M, Broecker V, Heratizadeh A (2008) Induction of chronic renal allograft injury by injection of a monoclonal antibody against a donor $\mathrm{MHC} \mathrm{lb}$ molecule in a nude rat model. Transpl Immunol 19: 187-191

8. Moraes JR, Stastny P (1977) A new antigen system expressed in human endothelial cells. J Clin Invest 60: 449-454

9. Papa ND, Raschi E, Moroni G (1999) Anti-endothelial cell lgG fractions from systemic lupus erythematosus patients bind to human endothelial cells and induce a pro-adhesive and a pro-inflammatory phenotype in vitro. Lupus 8: 423-429

10. Perrey C, Brenchley PE, Johnson RW, Martin S (1998) An association between antibodies specific for endothelial cells and renal transplant failure. Transpl Immunol 6: 101-106

11. Smith JD, Lawson C, Yacoub MH, Rose ML (2000) Activation of NF-kappa B in human endothelial cells induced by monoclonal and allospecific HLA antibodies. Int Immunol 12: 563-571

12. Solez K, Colvin RB, Racusen LC et al. (2008) Banff 07 classification of renal allograft pathology: updates and future directions. Am J Transplant 8: 753-760
13. Sumitran-Karuppan S, Tyden $G$, Reinholt F et al. (1997) Hyperacute rejections of two consecutive renal allografts and early loss of the third transplant caused by non-HLA antibodies specific for endothelial cells. Transpl Immunol 5: 321-327

14. Terasaki PI, Ozawa M, Castro R (2007) Four-year follow-up of a prospective trial of HLA and MICA antibodies on kidney graft survival. Am J Transplant 7: 408-415

15. Valujskikh A, Heeger PS (2003) Emerging roles of endothelial cells in transplant rejection. Curr Opin Immunol 15: 493-498

16. Vestweber D (2007) Adhesion and signaling molecules controlling the transmigration of leukocytes through endothelium. Immunol Rev 218: 178-196

17. Yard B, Spruyt-Gerritse M, Claas F et al. (1993) The clinical significance of allospecific antibodies against endothelial cells detected with an antibody-dependent cellular cytotoxicity assay for vascular rejection and graft loss after renal transplantation. Transplantation 55: 1287-1293

18. Zou Y, Stastny P, Susal C et al. (2007) Antibodies against MICA antigens and kidney-transplant rejection. N Engl J Med 357: 1293-300 